\title{
Airline Schedule Disruption Management The impact of flight delays on connection loss
}

\author{
Hicham Rahil $^{\text {** }}$ Badr Abou El Majd ${ }^{l}$ and Mohammed Bouchoum ${ }^{l}$ \\ ${ }^{1}$ LIMSAD, Hassan II University, Faculty of Science, Casablanca
}

\begin{abstract}
Air travel demand is important and many travellers choose to drive to larger airports instead of flying from a small airport for many reasons, especially availability of non-stop flights. Another reason is perceived reliability of service. Consultants have pointed to a large number of delays and cancellations as reasons for low passenger. However, the effect of these flight delays on actual travel times is less clear. Because connections are usually necessary when traveling from small airports, departure delays may lead to missed connections. In the case of a cancellation, need to wait several hours (often overnight) for the next flight due to the small number of daily departures. This paper evaluate the impact of delays and cancellations on the profit earned through the seats captured on new opened routes. This aspect of decision-making comes in the form of multi-objective problem by testing the impact of a new opened route in terms of flight delays costs, financial gain and the quality of the service provided to a target customer. The NSGA-II algorithm is adopted to generate a front of Paretooptimal compound of a number of optimal departure times to the new destination while ensuring the best fill rate, and a minimum flight delays. The experiences are based on the flights of the Royal Air Maroc Company on the Casablanca hub.
\end{abstract}

\section{Introduction}

In Air Transport field, the most important factor sought by the potential passenger is the shortness of the trip. Passengers therefore preferred the direct flights between the airport of origin and destination. When the passenger is obliged to carry out a correspondence, airlines have an incentive to attract these passengers to increase the profitability of their lines by providing a continuous service from airport of departure, passing via the connecting airport until reaching the final destination. Royal Air Morocco (RAM), the Morocco's flag carrier, hopes to take place among the major airlines pulling profits from the position of the Morocco being the Africa portal on Europe and well obviously at the air hub put at his disposal in Casablanca. In addition to its flights to more than 80 destinations, Royal Air Morocco now offers more flights around the world by many airline partners. An alliance would benefit the position of RAM to the Morocco, but also central Africa / Western, the largest international market of RAM after Europe. In this work, we are interested in the problem of insertion of the new departure and arrival times to the existing program of an airline, and we opted for multi-objective optimization to solve this problem, the multi objective optimization meets the need to satisfy conflicting requirements. Therefore, to travel from Casablanca to Agadir, we will seek to minimize the cost; the time spent and maximize comfort. The plane will be cheap, fast, and polluting while the car will be economic, long and low in pollutants. Finally, the train will be a compromise solution. As there are no better in every way than another solution, a different compromise according to persons must be chosen. The choice is subjective, and it is essential to provide the set of choices so do not exclude a possibility. Multi objective optimization is primarily a tool for decision support, and it is a person who will make the final decision.

\section{Outline of the paper}

This article will focus on the application of optimization algorithms evolutionary (EA) using air routes scheduling problems and provide a brief overview on how airlines use these techniques to develop and assess the decisions of schedule amendments required at the last minute. Section 2 of this document provides a mathematical formulation of the problem of adding new lines in an existing program, under specific constraints, cost and profitability associated with a given calendar change. Section 3 explains the optimization process to fix the problem. Section 4 will

\footnotetext{
* Corresponding author: hichamrahil@gmail.com
} 
provide experimental results that explain more and detailed model and the optimization process, discuss possible extensions of the other sketches and model of research. Section 5 concludes the paper and specifies certain points of view. At the end of the paper, we provide a complete list of references highlighted in the document.

\section{Methods}

\subsection{Problem Formulation}

A wide variety of problems in industry, and many other fields, involve the simultaneous optimization of several objectives. In many cases, the objectives are defined in incomparable units, and they present some degree of conflict among them (i.e., one objective cannot be improved without deterioration of at least another objective). These problems are called Multiobjective Optimization Problems (MOPs). Let us consider our problem of new route insertion in an existing flight program, where the air transport company is interested in minimizing the total duration of the generated delays over the hub to improve customer service. On the other hand, the company also wants to maximize the number captured seats in order to improve the market benefits. Clearly, these objectives are in conflict since capturing more seats increases benefits, but increases generated delays. In addition, the objectives of this problem are expressed in different measurement units. The method most commonly adopted in Multiobjective optimization to compare solutions is the one called Pareto dominance relation [1], which, instead of a single optimal solution, leads to a set of alternatives with different trade-offs among the objectives. These solutions are called Pareto optimal solutions or nondominated solutions. Although there are multiple Pareto optimal solutions, in practice, only one solution has to be selected for implementation. Therefore, in the Multiobjective optimization process we can distinguish two tasks, namely: i) find a set of Pareto optimal solutions, and ii) choose the most preferred solution out of this set. Since Pareto optimal solutions are mathematically equivalent, the latter task requires a Decision Maker (DM) who can provide subjective preference information to choose the best solution in a particular instance of the Multiobjective optimization problem. In this paper, the company wants to open a new destination, and the purpose is of course the conquest of new markets. Indeed, after the opening of a new air route on departure from its air hub, the company will seek to connect this new destination with other airports served by the same company. A study of the market is developed beforehand to set among airports served by the company, those that present important potential of passengers wishing to go to the new destination via a connecting flight through the hub of the company. Let us note by $x$ the departure time toward the new destination. Actually, $x$ is the decision variable of the problem, which will affect the two criteria considered in the problem. We will also note by " $t$ " the total duration of the flight to the new destination, and of course the value $x$ $+t$ is the scheduled return hour from the new destination.
We will limit our study to the 20 first airports ranked by origin-and-destination passenger volume and the busiest one is airport $k$ where $k=1$. However, decision-makers may extend targets considered according to demand and importance of the proposed destination. Let $A_{k}$ (resp. $D_{k}$ ) the set of the weekly arrivals time (resp. departures time) from an airport $k \in K(K=\{1,2, . ., 20)$ to the hub, where $\mathrm{K}$ is the set of twenty airports served weekly by RAM company and ranked by origin-and-destination passenger volume. $a_{k i}$ the ith arrival time (resp. $d_{k j}$ the jth departure time) with $i, j \in\left\{1,2, \ldots, N_{k}\right\} . \quad a_{k i}$ and $d_{k j}$ are ranked in ascending order), and $N_{k}$ is the weekly frequency of routes between hub and given airport $k \epsilon K$, we take the minute as unit of time. We note that, arrivals and departures time during the week are included in the interval [0, 10080].

$$
\begin{gathered}
\alpha_{k i}=\left\{\begin{array}{c}
1 \text { Outbound connection is realized for } a_{k i} \\
0 \text { Otherwise }
\end{array}\right. \\
\delta_{k j}=\left\{\begin{array}{c}
1 \text { Intbound connection is realized for } d_{k j} \\
0 \text { Otherwise }
\end{array}\right.
\end{gathered}
$$

$w_{k}$ are the weight vectors given by:

$w_{k}=\frac{P_{k}}{\sum_{i=1}^{20} P_{i}} \quad$ and $\quad \sum_{k=1}^{20} w_{k}=1$

$P_{k}$ is the origin-and-destination passenger volume recorded in a year between a given airport $k$ served by the RAM company and the new destination, $\sum_{i=1}^{20} P_{i}$ is the sum of the passenger volume of the twenty busiest air routes served by RAM, ranked by origin-and-destination passenger volume. This parameter is introduced to encourage the connection of airports with important passenger volume. $x \in\left[x+t+t_{\min }, 10080-t_{\max }\right]$, where $t_{\min }$ is the minimum connecting time, and $t_{\max }$ is the maximum allowed connecting time.

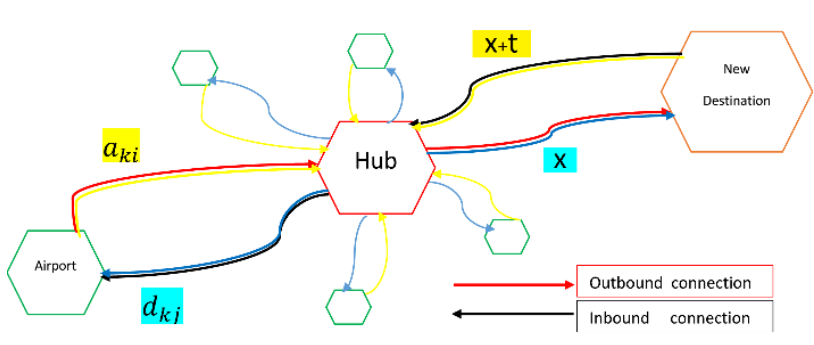

Fig. 1. The Inbound step, pass through the hub to join the new destination, and return for the outbound step.

Figure 1 illustrates the Outbound and Inbound connections, where the flight Inbound step (red color), pass through the hub to join the new destination, and return back again via the hub for the Outbound step (black color). During the optimization process, each random value $\mathrm{x}$ generates two intervals: $\left[x-t_{\max }, x-t_{\min }\right]$ and $\left[x+t+t_{\text {min }}\right.$ , $\left.x+t+t_{\max }\right]$. An Outbound connection is realized when a given value $a_{k i}$ comes inside Arrival time window [ $x$ - 
$\left.t_{\max }, x-t_{\min }\right],\left(\alpha_{k i}=1\right)$. In addition, the Inbound one is realized when a given value $d_{k j}$ is cached inside departure time window $\left[x+t+t_{\min }, x+t+t_{\max }\right],\left(\delta_{k j}=1\right)$.

STaki: is the actual time of occurrence of the estimated arrival $a_{k i}$;

$S T d_{k j}$ : is the actual time of occurrence of the estimated departure $d_{k j}$;

$C_{k i}$ : is the unit time delay cost of arrival aki ;

$C_{k j}$ : is the unit time delay cost of departure $d_{k j}$;

We note $X_{i j}^{k}=\alpha_{k i} \times \delta_{k j} \quad$ where

$X_{i j}^{k}=\left\{\begin{array}{c}1 \text { Full connection is realized for } a_{k i} \text { and } d_{k j} \\ 0 \text { Otherwise }\end{array}\right.$

\subsection{Multiobjective functions}

In order to find the best departure time $x$, we maximize the objective function $f_{1}$ and minimise $f_{2}$ :

$$
\begin{gathered}
f_{1}(x)=\sum_{k i j} w_{k} \times X_{i j}^{k} \\
f_{2}(x)=\sum_{k i j}\left[C_{k i} X_{i j}^{k}\left(S T a_{k i}-a_{k i}-\Delta \tau\right)\right. \\
\left.+C_{k j} X_{i j}^{k}\left(S T d_{k j}-d_{k j}-\Delta \tau\right)\right]
\end{gathered}
$$

A good choice of $x$, will enhance the value of the Objective function $f_{1}$ will be maximized with guaranteeing the connection in both directions, Outbound and Inbound through the factors $\alpha_{k i}$ multiplied by $\delta_{k j}$, and by promoting the best passenger volume through $\mathrm{w}_{\mathrm{k}}$. $\mathrm{f}_{1}$ is expressed through scalarization method, the weighted sum (WS) method. The WS method is the commonly used scalarization method because of its simplicity, ease of use, and direct translation of weight into the relative importance of the objectives [2]. Its drawbacks are also well known and discussed in literature [3]. These include the followings:

$\checkmark \quad$ It misses solution points on the non-convex part of the Pareto surface;

$\checkmark \quad$ Its diversity cannot be controlled, therefore even distribution of weights does not translate to uniform distribution of the solution points;

$\checkmark$ The distribution of solution points is highly dependent on the relative scaling of the objective.

For efficient results, the objective function $f_{2}$ calculates only the flight Delay Losses of connected flights. Thus the found results will help to quantify the impact of cumulated delays caused by these connections, and then will give us an idea about the resulting costs if we opt for this strategy. Finally, the minimization of $f_{2}$ will involve the reduction of the generated delays and will make subsequently the correspondence more comfortable for passengers, and therefore, will automatically reduce the costs related to passenger accommodation and all other related charges. $f_{2}$ is the Objective Function of Flight Delay Losses. In most cases, either the arrival or departure flights, the actual arrival time is almost impossible to be consistent with the estimated time, so we make the following provision. The following two situations are not treated as delay: (1) the actual time is earlier or later than the estimated time within $\Delta \boldsymbol{\tau}:$ (2) all the flights, which are in advance of the estimated time landing or taking off.

\section{Numerical Implementation}

\subsection{NSGA-II Algorithm}

The evolutionary optimization algorithm used to validate numerically the theoretical framework described above is called the Non-dominated Sorting Genetic Algorithm (NSGA-II) [4, 5]. NSGA-II uses a fast non-dominated sorting approach, with complexity $\mathrm{O}\left(\mathrm{MN}^{2}\right)$, an elitism approach, and uses the scaled crowding distance as a secondary diversity criterion, that requires no additional parameter, and figure 2 describes the NSGA-II procedure. All design variables here are continuous, and the variation operators have been chosen accordingly, as in described by Deb [6] . More precisely, all experiments use the simulated binary crossover operator (SBX) and the polynomial mutation, which can handle directly the box constraints. The other parameters of the algorithm are the population size, which is equal to the archive size, the probability of crossover, the probability of mutation, the dispersion coefficients, and the number of generations before termination. NSGA-II has been chosen here for its long record of successes, demonstrating its robustness to find a good approximation of the Pareto front. Further work will investigate whether other MOEAs outperform NSGA-II on this problem. A good candidate is SMPSO [7] that has excellent performances on continuous problems.

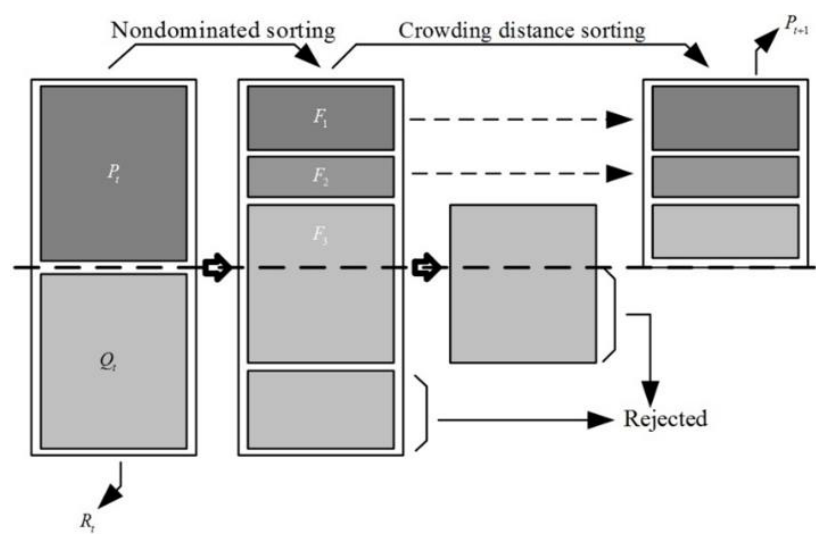

Fig. 2. NSGA-II procedure.

\subsection{Results and discussion}

Following the air carrier demand, we focus on the test of 20 airports; obtained results are given by figure 3 showing Pareto's front solutions with the corresponding 
coordinates. We obtain six optimal solutions, offering the possibility to connect 20-targeted airports to the new opening, maintaining an interesting fill rate and minimizing the stopover time over the hub.

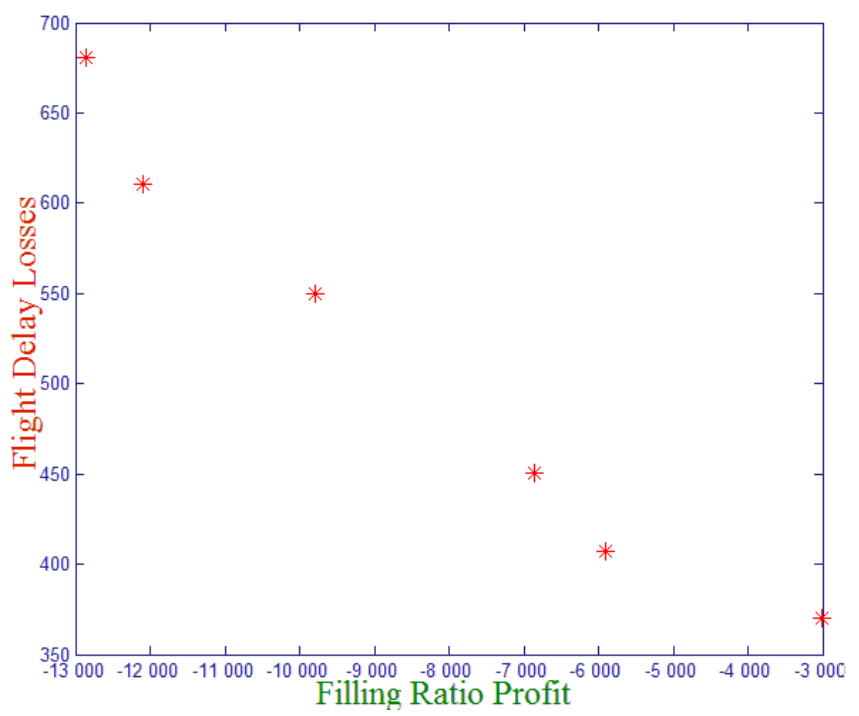

Fig. 3. Pareto optimals of twenty airports case.

Following the air carrier demand, we focus on the test of 20 airports; obtained results are given by figure 2 gives Pareto's front solutions with the corresponding coordinates. We obtain six optimal solutions, offering the possibility to connect 20-targeted airports to the new opening, maintaining an interesting fill rate and minimizing the effect of flight delays. Table 1 gives details of flight rotation corresponding to each solution from Pareto front. We notice that Airports ranked in following orders $15,16,17$, and 18 are not connected by the obtained solutions. $\mathrm{S}_{4}$ and $\mathrm{S}_{5}$ are very interesting solutions, connecting 12 (respect 11) of the 20 tested airports. Solution $\mathrm{S}_{4}$ provides $10 \mathrm{~h} 35 \mathrm{~min}$ maximum stopover time on the hub of Casablanca in outbound step, and less than one hour (55 minutes) as minimal stopover time. Departure time is Saturday at 06:30 UTC from Casablanca, the return is the same day at 23:00, and the inbound step is 1 hour 15 minutes stopover time in Casablanca and 08h20min maximum before continuing correspondences back to connected airports. Obtained Departures from Casablanca fits perfectly the fleet programming strategy adopted by Royal Air Maroc Company. Indeed, the company sends daily almost the two-thirds of its fleet on Africa within half an hour before midnight to return in the morning on the Casablanca hub before continuing to Europe, America and golf countries. Moreover, knowing that the best potentials of this example are African countries friends and allies of Morocco, the obtained arrival from the new opening around 23:00 (UTC) is perfectly convenient to the fleet departures to Africa starting around 23:30 (UTC). Similarly, a departure time toward the new opening at 06:30 (UTC) is a perfect knowing that the vast majority of those arrivals lands in Casablanca before 06:00 (UTC).
Table 1. $\left(f_{1} ; f_{2}\right)$ Solutions with correspondent Rotations

\begin{tabular}{|c|ll|l|}
\hline \multirow{2}{*}{$\begin{array}{c}\text { Optimal } \\
\text { Solutions }\end{array}$} & \multicolumn{3}{|c|}{ Flight Rotation (UTC) } \\
\cline { 2 - 4 } & \multicolumn{2}{|c|}{ Departure Time } & \multicolumn{1}{c|}{ Arrival Time } \\
\hline$S_{1}$ & Monday $-05: 00$ & Monday - 21:30 \\
$S_{2}$ & Thursday $-6: 40$ & Friday - 09:10 \\
$S_{3}$ & Thursday $-00: 50$ & Thursday - 06:40 \\
$S_{4}$ & Saturday $-06: 30$ & Saturday - 23:00 \\
$S_{5}$ & Tuesday $-06: 25$ & Tuesday - 22:55 \\
$S_{6}$ & Tuesday $-12: 05$ & Wednesday - 4:35 \\
\hline
\end{tabular}

\section{Conclusions and Future works}

Airline managers and decision makers in general, are probably interested to examine the results of this paper, allowing them a dynamic planning of their fleets without need to review the existing flight schedule. Indeed, when we take the decision to open a new destination, the reason may be political or purely commercial; we will be able with this model to insert this new program without losing scheduled connections and without causing major delays during the correspondences. We are all aware that the delay is a boring parameter and causes additional and significant costs for the airlines. Then this solution will serve as a tool to support the decision for an air carrier to amend its program and even think about increasing the number of frequencies on a specific route due to an unforeseen event without constraint to bring changes to the initial program. The Pareto front has been achieved using the algorithm of NSGA-II and the numerical results are based on the real case of the program of the Moroccan airline Royal Air Morocco (RAM) on the hub of Casablanca. Finally, we are all convinced that the aerospace industry is one of the most affected sectors by the hourly disruptions and delays due to several parameters. Airlines put and after developing, a program will face delays that spread quickly to connected flights, which induces considerably important and unexpected costs for airlines [8]. The primary goal now is to seek an effective way to minimize the impact of disruptions, reducing delays and the number of flights affected. In addition and for the perspective of this paper $[9,10]$, we will include the criterion of disruptions to introduce the aspect of robustness.

\section{References}

1. Pareto, Vilfredo. Cours d'économie politique. Vol. 1. Librairie Droz, (1964).

2. Marler, R. Timothy, and Jasbir S. Arora. "The weighted sum method for multi-objective ptimization: new insights." Structural and multidisciplinary optimization 41.6 (2010): 853-862. 
3. Das, Indraneel, and John E. Dennis. "A closer look at drawbacks of minimizing weighted sums of objectives for Pareto set generation in multicriteria optimization problems." Structural optimization 14.1 (1997): 63-69.

4. Kalyanmoy Deb, Multi-Objective Optimization using Evolutionary Algorithms, John Wiley and Sons, 5 juil. (2001) - 497 pages.

5. Zitzler, Eckart. "Evolutionary algorithms for multiobjective optimization: Methods and applications." (1999)

6. Deb, Kalyanmoy, et al. "A fast and elitist multiobjective genetic algorithm: NSGA-II." IEEE transactions on evolutionary computation 6.2 (2002): 182-197.

7. Kennedy, James. "Particle swarm optimization." Encyclopedia of machine learning. Springer US, (2011). 760-766.

8. Guimarans, Daniel, Pol Arias, and Miguel Mujica Mota. "Large Neighbourhood Search and Simulation for Disruption Management in the Airline Industry." Applied Simulation and Optimization. Springer International Publishing, (2015). 169-201.

9. Abou El Majd Badr, J.-A. Desideri, and A. Habbal, Aerodynamic and structural optimization of a business-jet wingshape by a Nash game and an adapted split of variables, Mec. Ind., vol. 11, no. 34, pp. 209-214.

10. Abou El Majd Badr and S. Kachroudi. A deterministic approach using game theory for multi-objective robust optimization, In the proceeding of the $3 \mathrm{rd}$ International Conference on Metaheuristics and Nature Inspired Computing, META'10, Djerba Island in Tunisia. 